ORIGINAL PAPER

\title{
The diagnostic utility of INSM 1 and GATA3 \\ IN DISCRIMINATING PROBLEMATIC MEDULLARY THYROID CARCINOMA, THYROID AND PARATHYROID LESIONS
}

\author{
Sarah Adel Hakim, Nermine Mohamed Abd Raboh
}

Department of Pathology, Faculty of Medicine, Ain Shams University, Cairo, Egypt

*These two authors equally contributed to this work.

\begin{abstract}
Discriminating thyroid and parathyroid lesions may sometimes pose a diagnostic difficulty. Medullary thyroid carcinomas (MTCs) display various cytologic and architectural features that resemble other thyroid and even rarely some parathyroid neoplasms. Moreover, some MTCs may have negative serum calcitonin, rendering them difficult to diagnose. Hence, to reach an appropriate diagnosis in problematic cases of these three categories - thyroid lesions, MTC and parathyroid lesions - the use of several immunohistochemical panels has been suggested and applied. However, conventional markers are not always conclusive in problematic cases. Thus, in the current study we aim to evaluate the diagnostic utility of using GATA3 and INSM1 (insulinoma-associated protein 1) as novel nuclear markers to be applied as an adjunct in case of histopathologic suspicion.

A retrospective study was carried out on samples of lesions from three groups: group 1: thyroid lesions (27), group 2: medullary thyroid carcinoma (25); 1/25 had negative serum levels of calcitonin, and group 3: parathyroid lesions (36). Biopsies were received at the Pathology Laboratory of Ain Shams University Hospitals.

INSM1 showed 98\% diagnostic accuracy in diagnosing MTC and differentiating it from other thyroid lesions. The case of MTC with negative serum calcitonin showed positive INSM1 staining. GATA3 showed $96.8 \%$ diagnostic accuracy in diagnosing parathyroid lesions and differentiating them from thyroid lesions.

Using immunohistochemical staining by GATA3 and INSM1, in the appropriate histopathological setting, significantly aids in the differentiation between thyroid lesions, parathyroid lesions and MTCs. INSM 1 could serve as a potential diagnostic marker in the rare cases of non-secretory MTC and in metastatic work up.
\end{abstract}

Key words: GATA3, INSM1, medullary carcinoma, thyroid, parathyroid.

\section{Introduction}

Parathyroid and thyroid lesions are common in routine histopathologic work. Normal parathyroid tissue is situated at the posterior or near the lower pole of the thyroid gland; hence most parathyroid lesions occur at this site [1]. Despite the fact that most parathyroid lesions have a straightforward histopathologic diagnosis, owing to their site in relation to the thyroid gland, some parathyroid lesions may pose a diagnostic difficulty in being discriminated from some thyroid lesions [2]. Medullary thyroid carcinoma (MTC), a well-differentiated neuroendocrine tumor of the thyroid gland, is derived from 
the parafollicular calcitonin-producing cells (C-cells). It constitutes $5-8 \%$ of all thyroid malignancies $[3,4]$, so due to the limited numbers of patients, its study is rather difficult. In addition, no agreement has been reached as regards the classification of its cytoarchitectural patterns [5]. Among the described subtypes are classical, papillary or pseudopapillary, glandular, giant cell, spindle cell, small cell, paraganglioma-like, clear cell, oncocytic, angiosarcoma-like, squamous cell and melanin producing subtypes $[6,7,8]$. Even mucus secretion has been observed in MTC [9]. The great variability in microscopic patterns of MTC, the presence of rare cases of non-secretory MTCs (i.e., not associated with elevated serum calcitonin), together with the possibility of metastatic lesions to the thyroid gland may render these cases difficult to diagnose and follow up $[10,11,12,13$, $14,15,16]$.

It is, therefore, mandatory to add new immunohistochemical markers as an adjunct to reach a proper diagnosis in problematic cases. Several immunohistochemical panels have been suggested and used, the majority of which are cytoplasmic, which may, in some instances, cause inaccurate evaluation of immunoreaction due to background staining [5]. On the other hand, transcription factors are highly specific, nuclear immunohistochemical (IHC) markers; among these are GATA3 and INSM1. GATA3 is an established marker of urothelial carcinoma [17] and primary breast carcinoma [18], with less expression in certain cases of metastatic breast carcinoma [19]. Moreover, GATA3 is involved in the embryonic development of parathyroid glands, as well as in adult parathyroid cell proliferation [2], while INSM1 is a relatively new and useful diagnostic marker of neuroendocrine differentiation [12, 20]. Thus, in the current study we aim to evaluate the diagnostic accuracy of using GATA3 and INSM1 (insulinoma-associated protein 1) as an adjunct in discriminating problematic cases within the spectrum of thyroid, parathyroid lesions and medullary thyroid carcinoma.

\section{Material and methods}

\section{Tissue and patient data}

The current study was a retrospective study conducted on 88 thyroid and parathyroid lesions that were obtained from the archives of the Pathology Laboratory of Ain Shams University Hospitals as a tertiary hospital in in Cairo, Egypt. These were received and diagnosed during the period from January 2017 to December 2019. Cases were obtained via total thyroidectomy. These 88 cases included 36 parathyroid lesions as follows: 15 parathyroid hyperplasia, 16 parathyroid adenoma and 5 cas- es of parathyroid carcinoma with prior clinical and radiological diagnosis, and 52 thyroid lesions as follows: 7 thyroid adenomas, 7 follicular carcinoma, 13 papillary carcinoma and 25 cases of medullary carcinoma. To simplify the statistical analysis, the 88 cases included in this study were divided based on their origin into three groups: group 1 - thyroid lesions (27); group 2 - medullary thyroid carcinoma (MTC) $(25 ; 1 / 25$ was non-secretory MTC with undetectable serum levels of calcitonin); and group 3 - parathyroid lesions (36). Hematoxylin and eosin-stained slides were examined to re-evaluate and verify the histopathologic diagnosis.

Inclusion criteria were as follows: a) all cases of MTCs, parathyroid and thyroid lesions received between January 2017 and December 2019 with available laboratory tests and whose diagnoses were confirmed by routine immunohistochemical tests, b) in the case of papillary thyroid carcinoma, only cases that posed diagnostic difficulty and necessitated confirmation via several immunohistochemical markers were included, c) only cases with enough tissue were selected in the analysis. Exclusion criteria: a) not enough tissue available, b) no available previous laboratory and radiological reports, c) straightforward papillary thyroid carcinoma.

Archival extraction of data about routine basic immunohistochemical markers, namely calcitonin, CEA (carcinoembryonic antigen) and PTH (parathyroid hormone), was done.

\section{Ethics statement}

All patients who participated in this study signed a written informed consent form before undergoing the surgery. The study was approved by the Research Ethical Committee (REC) at the Faculty of Medicine, Ain Shams University.

\section{Immunohistochemical staining}

Four micrometer sections of formalin-fixed and paraffin-embedded samples of thyroid, parathyroid and MTC samples were prepared. Immunohistochemical staining was performed using two primary antibodies on samples of the three groups; mouse monoclonal anti-GATA3 (Clone: HG3-31; Santa Cruz Biotechnology, Dallas, Texas, USA; 1: 100 dilution) and mouse monoclonal anti-INSM1 (Clone: A8; Santa Cruz Biotechnology, Dallas, Texas, USA; 1 : 200 dilution). Avidin-biotin immunoperoxidase complex technique was used according to Hsu et al. [21] by applying the Super Sensitive detection kit (Biogenex, Fermont, California, USA). The prepared tissue sections were fixed on poly-L-lysine coated slides overnight at $37^{\circ} \mathrm{C}$. Graded alcohol series were used to deparaffinize and rehydrate them. Then the sections were heated in a microwave oven 
in $10 \mathrm{mM}$ citrate buffer ( $\mathrm{pH}$ 6.0) for $20 \mathrm{~min}$. After the blocking of endogenous peroxidase and incubation in Protein Block Serum-Free Solution (Dako Cytomation, Glostrup, Denmark) for $20 \mathrm{~min}$, the sections were incubated overnight at $4^{\circ} \mathrm{C}$ with primary antibodies. Biotinylated anti-mouse immunoglobulin and streptavidin conjugated to horseradish peroxidase were then added. Finally, 3,3-diaminobenzidine as the substrate or chromogen was used to form an insoluble brown product. Finally, the sections were counterstained with hematoxylin and mounted. Sections of breast adenocarcinoma and normal parathyroid gland samples were used as a positive control for GATA3 and sections of non-neoplastic pancreatic tissue as a positive control for INSM1. Negative control sections of each lesion were incubated with normal mouse serum instead of each of the primary antibodies.

\section{Interpretation of immunohistochemical staining}

Immunohistochemical analysis of GATA3 and INSM1 was blindly performed by the two pathologists (the authors) without any prior knowledge of the clinicopathological data. Any discrepancies were resolved by consensus using a multi-headed microscope.

Nuclear staining of GATA3 and INSM1 in cells of any of the lesions of three groups was regarded as positive staining. Negative staining was defined as completely absent nuclear staining in the target cells.

No differences in the intensity of staining of target cells were detected in any of the 3 groups by any of the 2 IHC markers. Hence, the immunoreaction of both markers was graded according to the percentage of distinctly reactive cells $(0=$ negative $0 \%, 1+=$ focal $>0-25 \%$ and $2+=$ diffuse $>25 \%)$ [2, 22, 23].

\section{Statistical analysis}

Categorical variables are expressed as frequencies and percentages. Chi square and Fisher's exact test were used to examine the relationship between categorical variables. A significance level of $\mathrm{p}<0.05$ was used in all tests. All statistical procedures were carried out using SPSS version 15 for Windows (SPSS Inc., Chicago, IL, USA).

\section{Results}

Data for the 88 studied cases are represented in Table I.

\section{Immunohistochemical results}

The included adjacent normal parathyroid tissue showed no INSM1 expression, but expressed diffuse GATA3 nuclear expression; this staining served as
Table I. Data for the studied cases

\begin{tabular}{lcc}
\hline & N & $\%$ \\
\hline Groups & & \\
\hline Group 1: Thyroid lesions: & 27 & 30.7 \\
- Adenoma $(\mathrm{n}=7)$ & & \\
• Follicular carcinoma $(\mathrm{n}=7)$ & & \\
- Papillary carcinoma $(\mathrm{n}=13)$ & & \\
\hline $\begin{array}{l}\text { Group 2: Medullary carcinoma } \\
\text { (n }=25)\end{array}$ & 25 & 28.4 \\
\hline Group 3: Parathyroid lesions: & 36 & 40.9 \\
$\bullet$ Hyperplasia $(\mathrm{n}=15)$ & & \\
$\bullet$ Adenoma $(\mathrm{n}=16)$ & & \\
$\bullet$ Parathyroid carcinoma $(\mathrm{n}=5)$ & & \\
\hline GATA3 & & \\
\hline Negative & 54 & 61.4 \\
\hline Positive & 34 & 38.6 \\
\hline INSM1 & & \\
\hline Negative & 64 & 72.7 \\
\hline Positive & 24 & 27.3 \\
\hline
\end{tabular}

an internal positive control for GATA3. The included adjacent normal thyroid tissue showed neither INSM1 expression nor GATA3 expression. Thirtyfour $(94.4 \%)$ out of 36 cases of parathyroid lesions showed GATA3 nuclear expression. However, none of the parathyroid lesions showed INSM1 expression. None of the 25 cases of MTC showed GATA3 nuclear expression (Figs. 1, 2, 3).

Twenty-four (96\%) out of 25 cases of MTC showed INSM1 nuclear expression (Fig. 3). However, none of the 27 thyroid lesions showed INSM1 nuclear expression. On the other hand, none of the thyroid lesions or MTC cases showed GATA3 expression. There was a highly statistically significant difference between MTC and thyroid lesions as regards INSM1 IHC expression ( $\mathrm{p}=0.0001$; Table II). Among the 24 INSM1 positive MTC cases one was calcitonin-negative MTC (negative serum levels of calcitonin and negative calcitonin IHC).

Thirty-four (94.4\%) out of the 36 parathyroid lesions showed positive GATA3 nuclear expression (Figs. 1, 2). On the other hand, none of the thyroid lesions showed GATA3 expression. There was a highly statistically significant difference between parathyroid and thyroid lesions as regards GATA3 IHC expression ( $\mathrm{p}=0.0001$; Table III).

Concerning the grading of INSM1 immunoreaction in MTC cases, $76 \%$ of cases (19 out of 25 cases) showed $2+$ immunoreaction (Table IV).

Concerning the grading of GATA3 immunoreaction in different types of parathyroid lesions, there 

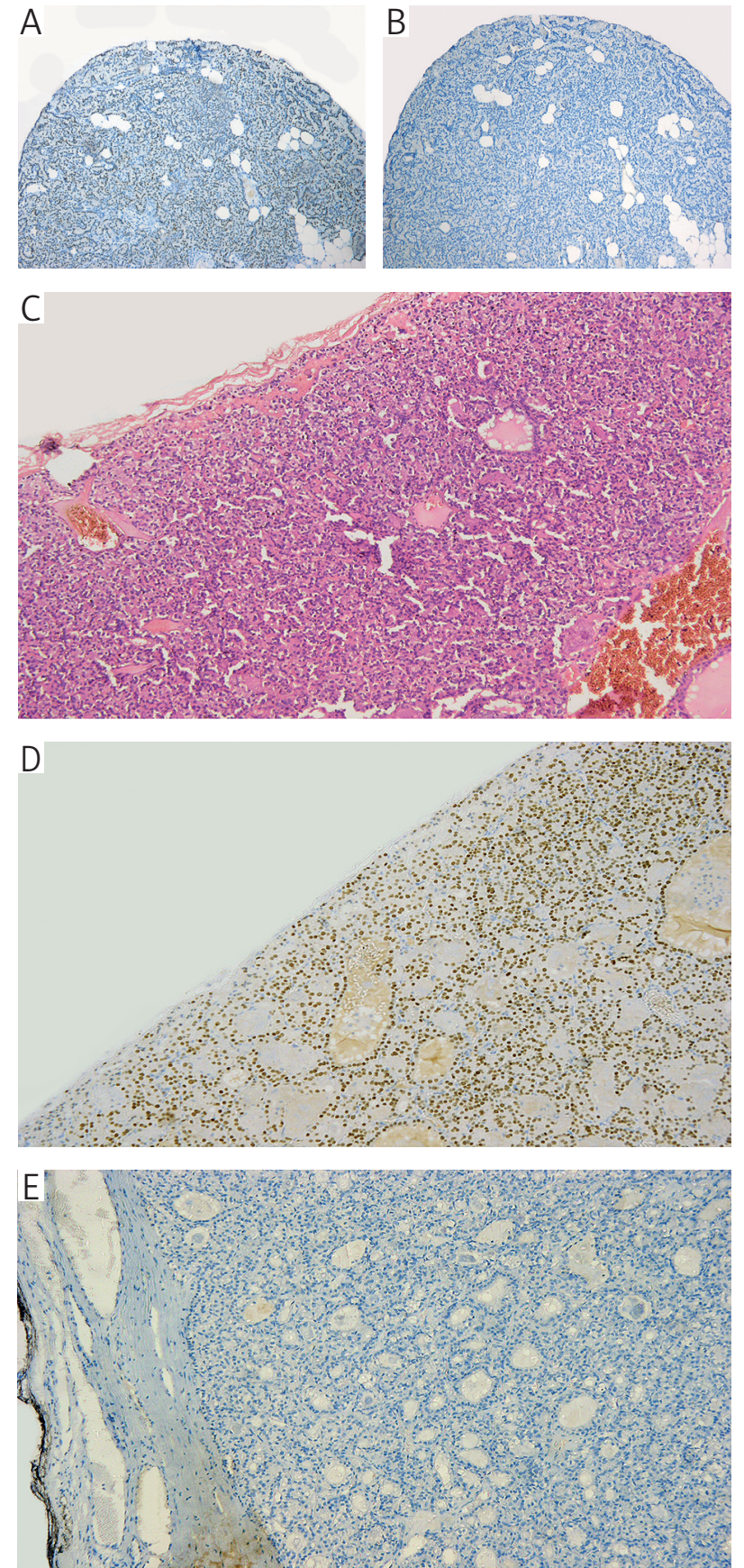

Fig. 1. Parathyroid hyperplasia GATA3 positive IHC, $100 \times(\mathrm{A})$; parathyroid hyperplasia INSM1 negative IHC, $100 \times(\mathrm{B}) ;$ parathyroid adenoma (encapsulated) HE, $40 \times(C)$; parathyroid adenoma GATA3 positive IHC, 100 $\times$ (D); parathyroid adenoma INSM1 negative IHC, $100 \times(\mathrm{E})$

was a highly significant difference between the type of parathyroid lesion and the grading of immunoreaction by GATA3; while all cases of parathyroid hyperplasia show the highest grading +2 , most cases of adenoma show +2 , while among carcinoma cases there are 2 cases with grade $+2,1$ case with grade +1 and 2 cases with no staining $(\mathrm{p}=0.025$; Table $\mathrm{V})$.

Upon using both studied markers in combination in problematic lesions within these three categories - thyroid lesions, MTCs and parathyroid lesions a highly statistically significant difference in expression of combined markers is noted, as $0 \%, 96 \%$ and $94.4 \%$ of thyroid lesions, MTCs and parathyroid lesions, respectively, show positivity for either of these two markers (Table VI).

In differentiating MTCs from thyroid lesions, combined markers show 96\%, 100\%, 100\%, $96.4 \%$ and $98 \%$ sensitivity, specificity, positive and negative predictive values (PPV, NPV) and accuracy, respectively. Moreover, in differentiating parathyroid from thyroid lesions, combined markers show $94.4 \%, 100 \%, 100 \%, 93.1 \%$ and $96.8 \%$ sensitivity, specificity, PPV, NPV and accuracy, respectively. On the other hand, in differentiating parathyroid lesions and MTCs, any of the two studied markers used singly would be sufficient, while combined use of both markers would be insignificant in this context (Table VII).

Concerning the conventional markers whose results are extracted from the archives of the $\mathrm{Pa}$ thology Laboratory, in differentiating MTCs from thyroid lesions, calcitonin shows $92.0 \%, 92.6 \%$, $92 \%, 92.5 \%$ and $92.3 \%$ sensitivity, specificity, PPV, NPV and accuracy respectively, while CEA showed $84.0 \%, 100 \%, 100 \%, 87 \%, 92.3 \%$ sensitivity, specificity, PPV, NPV and accuracy, respectively. Moreover, in differentiating parathyroid from thyroid lesions, PTH shows $86.1 \%, 100 \%$, $100 \%, 84.3 \%$ and $92 \%$ sensitivity, specificity, PPV, NPV and accuracy, respectively. On the other hand, in differentiating parathyroid lesions and MTCs, calcitonin shows $92.0 \%, 83.3 \%, 79.3 \%, 93.7 \%$ and $86.6 \%$ sensitivity, specificity, PPV, NPV and accuracy, respectively, while CEA showed $84.0 \%$, $100 \%, 100 \%, 90 \%, 93.4 \%$ sensitivity, specificity, PPV, NPV and accuracy, respectively. In differentiating parathyroid lesions from MTCs, PTH has $86.1 \%, 100 \%, 100 \%, 83.3 \%$, and $91.8 \%$ sensitivity, specificity, PPV, NPV and accuracy, respectively (Table VIII).

\section{Discussion}

MTCs display cytologic and architectural features that may resemble several primary and metastatic neoplasms [24]. Moreover, some parathyroid lesions are difficult for a pathologist to diagnose and distinguish from the neighboring thyroid lesions $[2,12]$. In addition, some features such as oncocytic changes are common findings in benign as well as malignant thyroid conditions $[25,26]$, and can pose diagnostic problems; for instance, the oncocytic cell type of papillary carcinoma is difficult to diagnose because it obscures the pattern of ground-glass nuclei [9]. Several studies aiming at refining important histopathological diagnostic parameters or combining immunohistochemical 

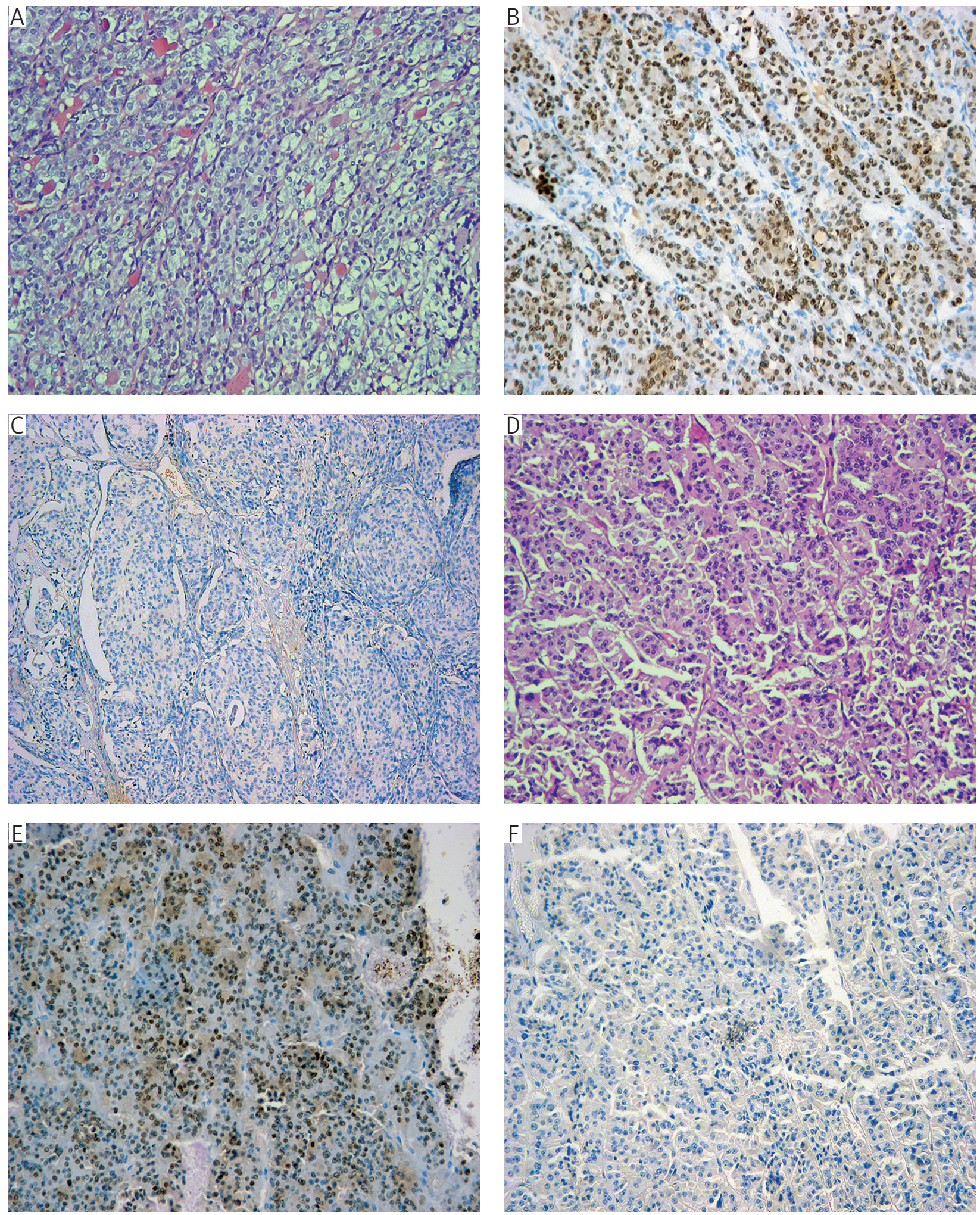

Fig. 2. Parathyroid carcinoma showing trabecular pattern HE, $200 \times($ A); parathyroid carcinoma GATA3 positive IHC, $200 \times(B)$; parathyroid carcinoma INSM1 negative IHC, $200 \times(C)$; parathyroid carcinoma oncocytic variant HE, 200× (D); parathyroid carcinoma, oncocytic variant, GATA3 positive IHC, $200 \times(\mathrm{E})$; parathyroid carcinoma, oncocytic variant, INSM1 negative IHC, $200 \times(\mathrm{F})$

markers have been conducted in this setting [27, 28]. Also, oncocytic parathyroid adenomas can resemble Hurthle cell tumor, and parathyroid lesions, in gener- al, may exhibit oxyphilic cells $[2,12]$. Therefore, IHC is strongly indicated for all cases of solid tumors without typical features of papillary or follicular carcinoma 

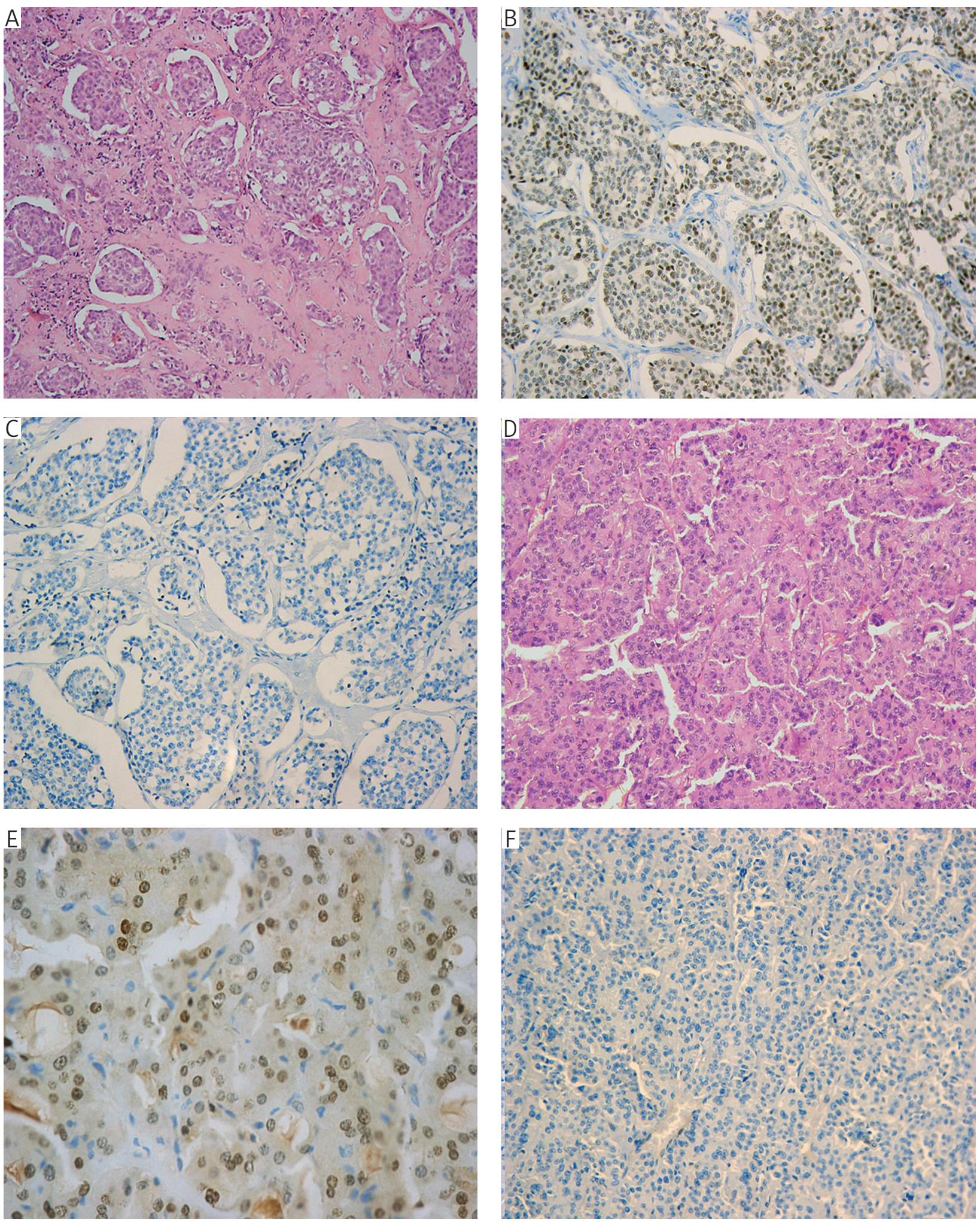

Fig. 3. MTC HE, $100 \times($ A), MTC INSM1 positive IHC, $100 \times(B)$; MTC GATA3 negative IHC, $100 \times(\mathrm{C})$; MTC oncocytic variant $\mathrm{HE}, 200 \times(\mathrm{D})$; MTC, oncocytic variant, INSM1 positive IHC, $400 \times(\mathrm{E})$; MTC, oncocytic variant, GATA3 negative IHC, $200 \times(\mathrm{F})$

to prevent underdiagnosis of medullary carcinoma and for proper assessment of these intricate lesions [9].

The conventional markers are not always conclusive; thyroid transcription factor-1 (TTF-1) is posi- tive in $96 \%$ of papillary carcinomas, $100 \%$ of follicular carcinomas, $20 \%$ of oncocytic tumors and $90 \%$ of MTCs, whereas anaplastic carcinomas are essentially immunonegative for TTF-1 [29]. Ninety-five 
Table II. Comparison between medullary carcinoma and thyroid lesions as regard the results of studied markers

\begin{tabular}{|c|c|c|c|c|c|c|}
\hline & \multicolumn{4}{|c|}{ GROUP } & \multirow[t]{3}{*}{$\mathbf{P}$} & \multirow[t]{3}{*}{ SIG } \\
\hline & \multicolumn{2}{|c|}{ MEDULLARY CARCINOMA } & \multicolumn{2}{|c|}{ THYROID LESIONS } & & \\
\hline & $\mathbf{N}$ & $\%$ & $\mathrm{~N}$ & $\%$ & & \\
\hline \multicolumn{7}{|l|}{ GATA3 } \\
\hline Positive & 0 & 0.0 & 0 & 0.0 & \multirow[t]{2}{*}{$0.0001^{*}$} & \multirow[t]{2}{*}{ HS } \\
\hline Negative & 25 & 100.0 & 27 & 100.0 & & \\
\hline \multicolumn{7}{|l|}{ INSM1 } \\
\hline Positive & 24 & 96.0 & 0 & 0.0 & \multirow[t]{2}{*}{-----} & \multirow[t]{2}{*}{----} \\
\hline Negative & 1 & 4.0 & 27 & 100.0 & & \\
\hline
\end{tabular}

Table III. Comparison between parathyroid and thyroid lesions as regard the results of studied markers (INSM1 and GATA3)

\begin{tabular}{|c|c|c|c|c|c|c|}
\hline & \multicolumn{4}{|c|}{ GROUP } & \multirow[t]{3}{*}{$\mathrm{P}$} & \multirow[t]{3}{*}{ SIG } \\
\hline & \multicolumn{2}{|c|}{ PARATHYROID LESION } & \multicolumn{2}{|c|}{ THYROID LESIONS } & & \\
\hline & $\mathbf{N}$ & $\%$ & $\mathbf{N}$ & $\%$ & & \\
\hline \multicolumn{7}{|l|}{ GATA3 } \\
\hline Positive & 34 & 94.4 & 0 & 0.0 & \multirow[t]{2}{*}{$0.0001 *$} & \multirow[t]{2}{*}{ HS } \\
\hline Negative & 2 & 5.6 & 27 & 100.0 & & \\
\hline \multicolumn{7}{|l|}{ INSM1 } \\
\hline Positive & 0 & 0.0 & 0 & 0.0 & \multirow[t]{2}{*}{-} & \multirow[t]{2}{*}{-} \\
\hline Negative & 36 & 100.0 & 27 & 100.0 & & \\
\hline
\end{tabular}

percent of medullary carcinomas are positive for calcitonin [9]. Previous studies have shown that although calcitonin is highly specific for MTC, the staining pattern may vary; it is usually diffuse, but can be focal (with $25 \%$ or less of cells exhibiting cytoplasmic reactivity) [30]. Absent calcitonin expression could occur in up to $5 \%$ of MTC cases [31]. As an alternative, in such cases where conventional immunohistochemical markers are inconclusive, in situ hybridization would be used for detecting calcitonin and calcitonin gene-related peptide mRNAs, but this technique is both expensive and is not always available in all institutes [32, 33]. In MTCs that show no elevation in serum calcitonin, the diagnosis may be delayed, which may worsen the condition. Even at the tissue level, only less than half of these cases show focal or diffuse IHC expression of calcitonin [10]. Therefore, finding other alternative immunohistochemical markers that help in identifying MTC and differentiating it from other thyroid lesions/tumors is mandatory in such cases. CEA has a role in diagnosing such cases [14], but being a cytoplasmic marker, CEA immunostaining in thyroid has been described to have non-specific reaction with CEA-like substances, a problem minimized by the advent of monoclonal CEA, and usually
Table IV. Grading of staining of INSM1 in MTC (medullary thyroid carcinoma) cases

\begin{tabular}{lcc}
\hline & $\mathbf{N}$ & $\%$ \\
\hline INSM1 & & \\
\hline Negative & 1 & 4.0 \\
\hline+1 & 5 & 20.0 \\
\hline+2 & 19 & 76.0 \\
\hline
\end{tabular}

suggests the diagnosis of MTC, but could not exclude other lesions such as thymic-derived lesions or metastatic carcinoma [9, 34, 35, 36]. Although metastasis to the thyroid is rarely encountered, the possibility of a metastatic deposit should be excluded in any suspicious lesion of the thyroid, owing to its poor prognosis $[37,38]$. Metastatic deposits in the thyroid could be mucinous in nature in cases of ovarian or lung carcinoma. This can lead to a difficulty in diagnosing the mucous-secreting variant of MTC. CEA will not solve this diagnostic dilemma, being positive in both cases. Thus, the need of other adjunct markers to be added the conventional panel remains. INSM1 by contrast will stain positive for mucous se- 
Table V. Grading of staining of GATA3 according to types of parathyroid lesions

\begin{tabular}{|c|c|c|c|c|c|c|c|c|}
\hline & \multicolumn{6}{|c|}{ PARATHYRoId LESIONS } & \multirow[t]{3}{*}{$\mathbf{P}$} & \multirow[t]{3}{*}{ SIG } \\
\hline & \multicolumn{2}{|c|}{ HYPERPLASIA } & \multicolumn{2}{|c|}{ ADENOMA } & \multicolumn{2}{|c|}{ CARCINOMA } & & \\
\hline & $\mathbf{N}$ & $\%$ & $\mathbf{N}$ & $\%$ & $\mathrm{~N}$ & $\%$ & & \\
\hline \multicolumn{9}{|c|}{ Intensity of GATA3 staining } \\
\hline 0 & 0 & 0.0 & 0 & 0.0 & 2 & 40.0 & \multirow[t]{3}{*}{$0.025 * *$} & \multirow[t]{3}{*}{$\mathrm{S}$} \\
\hline+1 & 1 & 6.7 & 3 & 18.8 & 1 & 20.0 & & \\
\hline+2 & 14 & 93.3 & 13 & 81.3 & 2 & 40.0 & & \\
\hline
\end{tabular}

Table VI. Description and comparison of combined markers (any positive GATA3 and/or INSM1) between the 3 studied groups

\begin{tabular}{|c|c|c|c|c|c|c|c|c|}
\hline & \multicolumn{6}{|c|}{ GROUP } & \multirow[t]{3}{*}{$\mathrm{P} *$} & \multirow[t]{3}{*}{ SIGNIFICANCE } \\
\hline & \multicolumn{2}{|c|}{ THYROID LESIONS } & \multicolumn{2}{|c|}{ MEDULLARY CARCINOMA } & \multicolumn{2}{|c|}{ PARATHYRoId LESIONS } & & \\
\hline & $\mathrm{N}$ & $\%$ & $\mathrm{~N}$ & $\%$ & $\mathrm{~N}$ & $\%$ & & \\
\hline \multicolumn{9}{|c|}{ INSM1/GATA3 (any positive marker) } \\
\hline Negative & 27 & 100.0 & 1 & 4.0 & 2 & 5.6 & \multirow[t]{2}{*}{0.001} & \multirow[t]{2}{*}{ HS } \\
\hline Positive & 0 & 0.0 & 24 & 96.0 & 34 & 94.4 & & \\
\hline
\end{tabular}

Table VII. Diagnostic criteria of combined studied markers (INSM1 and GATA3) in differentiating the three studied groups

\begin{tabular}{lccccc}
\hline & SENSITIVITY & SPECIFICITY & PPV & NPV & ACCURACY \\
\hline MTC and thyroid lesions & $96.0 \%$ & $100.0 \%$ & $100 \%$ & $96.4 \%$ & $98 \%$ \\
\hline Parathyroid and thyroid lesions & $94.4 \%$ & $100.0 \%$ & $100 \%$ & $93.1 \%$ & $96.8 \%$ \\
\hline MTC and parathyroid lesions & $94.4 \%$ & $4 \%$ & $58.6 \%$ & $33.3 \%$ & $57.3 \%$ \\
\hline
\end{tabular}

Table VIII. Detailed diagnostic criteria of conventional markers (calcitonin, CEA and PTH) in differentiating the studied groups

\begin{tabular}{|c|c|c|c|c|c|}
\hline & Sensitivity $(\%)$ & SPECIFICITY (\%) & PPV (\%) & NPV (\%) & ACCURACY (\%) \\
\hline Calcitonin & 92.0 & 92.6 & 92 & 92.5 & 92.3 \\
\hline CEA & 84.0 & 100.0 & 100.0 & 87 & 92.3 \\
\hline PTH & 0 & 100.0 & 0 & 51.9 & 51.9 \\
\hline \multicolumn{6}{|c|}{ DiAGNOSTIC ACCURACY CRITERIA FOR CALCITONIN, CEA, AND PTH IN DIFFERENTIATING PARATHYROID FROM THYROID LESIONS } \\
\hline & SENSITIVITY (\%) & SPECIFICITY $(\%)$ & PPV (\%) & NPV (\%) & ACCURACY $(\%)$ \\
\hline Calcitonin & 16.7 & 92.6 & 75 & 45.4 & 49.2 \\
\hline CEA & 0 & 100.0 & 0 & 42.8 & 42.8 \\
\hline PTH & 86.1 & 100.0 & 100 & 84.3 & 92 \\
\hline \multicolumn{6}{|c|}{ DiAGNOSTIC ACCURACY CRITERIA FOR CALCITONIN, CEA, AND PTH IN DIFFERENTIATING MTC AND PARATHYROID LESIONS } \\
\hline & SENSITIVITY $(\%)$ & SPECIFICITY $(\%)$ & PPV (\%) & NPV $(\%)$ & ACCURACY $(\%)$ \\
\hline Calcitonin & 92.0 & 83.3 & 79.3 & 93.7 & 86.6 \\
\hline CEA & 84.0 & 100.0 & 100.0 & 90 & 93.4 \\
\hline PTH & 86.1 & 100.0 & 100.0 & 83.3 & 91.8 \\
\hline
\end{tabular}


creting MTC, but not metastatic ovarian and lung adenocarcinomas [39].

Some of the above-mentioned problems were encountered among MTC cases in the current work. For diagnosing MTC from thyroid lesions, calcitonin showed 92.0\%, 92.6\%, 92\%, 92.5\% and $92.3 \%$ sensitivity, specificity, PPV, NPV and accuracy, respectively, while CEA showed $84.0 \%, 100 \%, 100 \%$, $87 \%, 92.3 \%$ sensitivity, specificity, PPV, NPV and accuracy, respectively. Moreover, in differentiating parathyroid lesions and MTCs, calcitonin shows $92.0 \%, 83.3 \%, 79.3 \%, 93.7 \%$ and $86.6 \%$ sensitivity, specificity, PPV, NPV and accuracy, respectively, while CEA showed 84.0\%, 100\%, 100\%, 90\%, 93.4\% sensitivity, specificity, PPV, NPV and accuracy, respectively. Thus, these conventional markers yielded high diagnostic accuracy among cases of the current work, yet in problematic cases, INSM1 was able to provide a diagnostic aid in certain problematic cases, whereas calcitonin and CEA were not able to do so. For instance, one case of MTC had negative serum calcitonin. Moreover, it showed negative immunohistochemical expression of calcitonin at the tissue level. To establish the diagnosis of MTC, in situ hybridization was performed in a private laboratory outside our hospital to detect calcitonin and was a costly and time-consuming procedure. This case showed INSM1 positivity and GATA3 negativity in the current work. This provides a low-cost ancillary diagnostic aid if validated by further studies with a larger cohort.

Among the technical problems that we faced in some cases was the tendency of calcitonin to show only focal cytoplasmic expression of some tumor cells. Moreover, two of the cases included in the current work had a history of lung adenocarcinoma, so we could not exclude the remote possibility of the lesions being metastatic tumors to the thyroid gland and not MTCs, since CEA would be positive in both cases. INSM1, on the other hand, is only positive in the case of MTC. Thus, adding INSM1 as an adjunct to traditional markers in problematic cases would be of great help.

Previous studies have shown that calcitonin is expressed in about $15 \%$ of parathyroid hyperplasia cases, $23 \%$ of parathyroid adenomas, whereas all normal parathyroid glands were negative [40]. Several studies stated that parathyroid carcinoma cases were negative for both TTF-1 and calcitonin [41]. However, Hodgson et al. [42] presented a case of parathyroid carcinoma expressing immunohistochemical positivity to calcitonin. The rare intrathyroid location of parathyroid lesions could pose a diagnostic difficulty in their discrimination from benign follicular lesions of the thyroid [43]. Parathyroid lesions/tumors are usually positive for parathyroid hormone (PTH). This is in line with the results of PTH in the current work, which showed high diagnostic accuracy in differentiating parathyroid lesions from MTCs and from other thyroid lesions. However, like most hormones PTH can be aberrantly expressed in other tumors; therefore, many markers continue to be evaluated for diagnostic utility [44, 45].

Concerning GATA3 IHC expression in parathyroid lesions included in the current study, 34 out of the 36 cases of parathyroid lesions $(94.4 \%)$ showed positive GATA3 nuclear staining. This was less than what was recorded by Takada et al. [1], who stated that 66 out of their 67 parathyroid lesions (98.5\%) showed positive nuclear staining. This discrepancy could be attributed to different sample sizes, different parathyroid lesions in their study, which did not include parathyroid carcinoma cases, and different nature of specimens, their samples being cytological.

In the current study all cases of parathyroid hyperplasia (15 cases) showed positive nuclear GATA3 staining, among which 14 cases $(93.3 \%)$ showed diffuse $(+2)$ immunoreaction, while one case showed focal $(+1)$ immunoreaction. All cases (16 cases) of parathyroid adenoma showed positive GATA3 nuclear staining; 13 out of the 16 cases $(81.3 \%)$ showed +2 staining, while 3 out of the 16 cases (18.8\%) showed +1 staining. Three out of 5 parathyroid carcinoma cases showed positive nuclear staining (60\%), 2 cases of which showed +2 staining $(40 \%)$ and 1 case showed +1 staining $(20 \%)$. This was to some extent higher than the results stated by Ordonez [2], where GATA3 immunoreaction was also graded according to the percentage of positive cells, but into 4 grades (unlike the 2 -tier grading system we adopted in the current study); $80 \%$ of parathyroid hyperplasia cases, $72.7 \%$ of parathyroid adenoma cases and $33.3 \%$ of carcinoma cases showed the highest immunoreaction grading. This difference could be attributed to 2-tier versus 4-tier grading systems. On the other hand, our results were lower than those of Betts et al. [46], who had all their 7 parathyroid cases only showing strong nuclear staining, which could be attributed to their small sample size.

An important differential diagnosis in this context is to differentiate MTC from paraganglioma of the head and neck owing to its different management from that of other NETs. A subtype of paraganglioma that is mostly encountered in the head and neck region is the non-functioning paraganglioma. This subset shows focal-to-weak or even absent staining for tyrosine hydroxylase, together with keratin negativity. In this specific context, positivity for GATA3 may help to support a diagnosis of paraganglioma. In addition, absent staining of $\mathrm{PTH}$, calcitonin and CEA is also needed to diagnose "cytokeratin negative, tyrosine hydroxylase negative" paraganglioma in the head and neck region so as to exclude other lesions such as parathyroid neoplasms, 
which are also positive for GATA3 [47]. Furthermore, Rosenbaum et al. demonstrated that INSM1 would also be positive for paraganglioma [39]. This could be a useful asset that could be provided by the combination of both INSM1 and GATA3 in this context.

Concerning INSM1 IHC expression in parathyroid lesions included in the current study, all of the parathyroid lesions (36 cases) showed negative INSM1 IHC expression. This is in line with the results of Rooper et al. [20], Yu et al. [23] and Rosenbaum et al. [39], all of their parathyroid lesions/tumors having showed negative INSM1 IHC staining.

In the present study, 24 out of 25 MTC cases (96\%) showed INSM1 nuclear expression. This was in agreement with Rosenbaum et al. [39], who observed INSM1 nuclear expression in $96 \%$ of their cases. In contrast, none of the MTC cases showed GATA3 expression (0\%). This complemented the findings of Ordonez [2], none of whose MTC cases showed GATA3 expression.

Concerning grading of INSM1 immunoreaction, 19 out of 25 MTC cases (76\%) in the present study showed diffuse $(+2)$ nuclear staining, while 5 cases $(20 \%)$ showed focal $(+1)$ expression. This is to some extent like the findings of Rooper et al. [20], whose positive MTC cases, in terms of proportion of positive tumor cells, showed a mean of $86 \%$, thus showing diffuse staining.

Concerning the thyroid lesions in the current study, all cases (27) were negative for both GATA3 and INSM1. This was in agreement with studies by Ordonez [2] and Betts et al. [46], in which none of the thyroid lesions/tumors showed GATA3 expression. Our results also agree with those of Rosenbaum et al. [39], in which none of the thyroid lesions/tumors (excluding MTC) expressed INSM1.

In the current study, the adjacent normal thyroid tissue was negative for both INSM1 and GATA3, which corroborates the findings of Rosenbaum et al. [39], who stated that INSM1 was not expressed in normal thyroid tissue in their study. Also, Miettinen et al. [48], demonstrated GATA3 negativity in normal thyroid tissue. On the other hand, the adjacent normal parathyroid tissue in the current study was positive for GATA3, but negative for INSM1. This was in agreement with Ordonez [2]; all of the normal parathyroid tissue included in his study showed nuclear positivity for GATA3. Moreover, Yu et al. [23] observed INSM1 negativity in normal parathyroid tissue.

Unlike hormones, developmental transcription factors are rarely aberrantly expressed in well-differentiated neuroendocrine neoplasms. Transcription factors are highly specific IHC markers; among these are GATA3 and INSM1. It is worth noting that since both of the studied markers are transcription factors, their nuclear localization offers better evaluation of immunoreaction, with low background staining, unlike most of the conventional cytoplasmic markers used in this scope $[23,39]$. Therefore, in the current study we suggested the use of GATA3 and INSM1 IHC for better differentiation of difficult cases in the spectrum of thyroid, MTC and parathyroid lesions/tumors.

In case of a problematic lesion that falls into the category of thyroid/ parathyroid/ MTC, and guided by the results of the current study, GATA3 and INSM1 IHC markers could be added to the panel of markers, where GATA3 showed a highly statistically significant relation $(\mathrm{p}=0.0001)$ in differentiating parathyroid from thyroid lesions, with diagnostic accuracy, sensitivity, specificity, PPV and NPV of $96.8 \%, 94.4 \%, 100 \%, 100 \%$ and $93.1 \%$, respectively. The current study also demonstrated that in discriminating MTC from other thyroid lesions, INSM1 showed a highly statistically significant relation ( $\mathrm{p}=0.0001)$, with diagnostic accuracy, sensitivity, specificity, PPV and NPV of 98\%, 96\%, $100 \%, 100 \%$ and $96.4 \%$, respectively.

In the same context, Ordonez [2] demonstrated GATA3 positivity in all of his parathyroid lesions and he stated that GATA3 is a very sensitive parathyroid marker. Also, Betts et al. [46] showed that GATA3 had $100 \%$ sensitivity and $97 \%$ specificity in discriminating parathyroid lesions from thyroid lesions. The discrepancy in their results as compared to ours may be attributed to our smaller sample size and the different subtypes included in the two studies. According to Rooper et al. [20], INSM1 was positive in all types of head and neck neuroendocrine tumors evaluated in their study (including MTC) with 99\% sensitivity. Moreover, INSM1 was negative in almost all non-neuroendocrine head and neck tumors including thyroid and parathyroid lesions (97.6\% specificity). The discrepancy might be attributed to the fact that, unlike our study, their study comprised head and neck lesions other than thyroid and parathyroid lesions.

The current study showed a highly statistically significant relation between the two markers in differentiating the three studied categories. Nevertheless, INSM1 could serve (alone without GATA3) as a diagnostic marker to differentiate MTCs from thyroid lesions and from parathyroid lesions. In fact, a recent study has claimed that INSM1 was sufficiently sensitive and specific to serve as a standalone marker for neuroendocrine tumors of the head and neck [49].

The usual technique for diagnosing a neuroendocrine tumor is through a panel approach. Combining monoclonal antibodies with transcription factors, hormones and cell-specific peptides often assists diagnosticians in the assessment of cellular origin of a neuroendocrine neoplasm [50]. In the current study, we tested the diagnostic accuracy of two 
transcription factors, and guided by our results, we concluded that using immunohistochemical staining by GATA3 and INSM1 can significantly aid in the differentiation between thyroid lesions, parathyroid lesions and MTCs in problematic cases. Furthermore, INSM1 may serve as a complementary marker for the diagnosis of non-secretory and metastatic MTCs, after validating our findings in further studies with larger sample sizes. A limitation to this study is the small number of cases of some thyroid lesions, MTCs and of parathyroid carcinomas, so further studies are needed to validate a panel of immunohistochemical markers that prove most useful as an adjunct in the evaluation of challenging MTCs, thyroid, parathyroid lesions/tumors.

\section{The authors declare no conflict o interest.}

\section{References}

1. Takada N, Hirokawa M, Suzuki A, et al. Diagnostic value of GATA-3 in cytological identification of parathyroid tissues. Endocr J 2016; 63: 621-626.

2. Ordóñez NG .Value of GATA3 immunostaining in the diagnosis of parathyroid tumors. Appl Immunohistochem $\mathrm{Mol}$ Morphol 2014; 22: 756-761.

3. Somnay YR, Schneider D, Mazeh H. Thyroid: medullary carcinoma. Atlas Genet Cytogenet Oncol Haematol 2013; 17 291-296.

4. Greco C, Brigante G, Taliani E, et al. Concomitant medullary thyroid carcinoma with paraganglioma-like pattern and papillary thyroid carcinoma. Endocrinol Diabetes Metab Case Rep 2019; 2019: 19-0094.

5. Ríos A, Rodríguez JM, Febrero B, et al. Histological and immunohistochemical profile of sporadic and familial medullary thyroid carcinoma. Endocrinol Nutr 2011; 58: 521-528.

6. Vinciguerra GLR, Noccioli N, Cippitelli C, et al. Oncocytic variant of medullary thyroid carcinoma: a rare case of sporadic multifocal and bilateral RET wild-type neoplasm with revision of the literature. Rare Tumors 2016; 8: 6537.

7. Singh K, Sharma MC, Jain D, Kumar R. Melanotic medullary carcinoma of thyroid - report of a rare case with brief review of literature. Diagn Pathol 2008; 3: 2; doi:10.1186/1746-1596-3-2.

8. Wang C, Yun T, Wang Z, et al. Pathological characteristics and genetic features of melanin-producing medullary thyroid carcinoma. Diagn Pathol 2018; 13: 86; doi: 10.1186/s13000018-0764-2.

9. Tatic S. Histopathological and immunohistochemical features of thyroid carcinoma. Arch Oncol 2003; 11: 173-174.

10. Gambardella C, Offi C, Patrone R, et al. Clinical challenges with calcitonin-negative medullary thyroid carcinoma. J Cancer Res Clin Oncol 2016; 142: 2023-2029.

11. Alapat DV, Ain KB, Sloan DA, et al. Disparity between tissue and serum calcitonin and carcinoembryonic antigen in a patient with medullary thyroid carcinoma. Endocrine 2011; 39: 148-152.

12. Giovanella L, Crippa S, Cariani L. Serum calcitonin-negative medullary thyroid carcinoma: role of $\mathrm{CgA}$ and CEA as complementary markers. Int J Biol Markers 2008; 23: 129-131.

13. Frank-Raue K, Machens A, Leidig-Bruckner G, et al. Prevalence and clinical spectrum of nonsecretory medullary thyroid carcinoma in a series of 839 patients with sporadic medullary thyroid carcinoma. Thyroid 2013; 23: 294-300.
14. Moo-Young TA, Traugott AL, Moley JF. Sporadic and Familial Medullary Thyroid Carcinoma: State of the Art. Surgical Clinics 2009; 89: 1193-1204.

15. Scollo C, Baudin E, Travagli JP, et al. Rationale for central and bilateral lymph node dissection in sporadic and hereditary medullary thyroid cancer. J Clin Endocrinol Metab 2003; 88: 2070-2075.

16. Liang Y, Heitzman J, Kamat AM, et al. Differential expression of GATA-3 in urothelial carcinoma variants. Hum Pathol 2014; 45: 1466-1472.

17. Shaoxian T, Baohua Y, Xiaoli X, et al. Characterisation of GATA3 expression in invasive breast cancer: differences in histological subtypes and immunohistochemically defined molecular subtypes. J Clin Pathol 2017; 70: 926-934.

18. Stolnicu S, Tunde C, Cadar A, Boros M. Differences in GATA3 expression among histological/molecular subtypes and grades in infiltrating breast carcinoma (IBC) are important in the diagnosis of metastatic breast carcinoma. Pol J Pathol 2020; 71 : 62-65.

19. Rooper LM, Bishop JA, Westra WH. INSM1 is a sensitive and specific marker of neuroendocrine differentiation in head and neck tumors. Am J Surg Pathol 2018; 42: 665-671.

20. Hsu SM, Raine L, Fanger H. Use of avidin-biotin-peroxidase complex $(\mathrm{ABC})$ in immunoperoxidase techniques: a comparison between $\mathrm{ABC}$ and unlabelled antibody (PAP) procedures. J Histochem Cytochem 1981; 29: 577-580.

21. Turchini J, Sioson L, Clarkson A, et al. Utility of GATA-3 expression in the analysis of pituitary neuroendocrine tumour (PitNET) transcription factors. Endocr Pathol 2020; 31: 150155 .

22. Yu Q, Hardin H, Chu YH, et al. Parathyroid neoplasms: immunohistochemical characterization and long noncoding RNA (lncRNA) expression. Endocr Pathol 2019; 30: 96-105.

23. Gucer H, Caliskan S, Kefeli M, Mete O. Do you know the details of Your PAX8 antibody? monoclonal PAX8 (MRQ-50) is not expressed in a series of 45 medullary thyroid carcinomas. Endocr Pathol 2020; 31: 33-38.

24. Patrizia S, Giuseppe S, Francesco P, et al. Cytologic diagnosis of oncocytic neoplasms of the thyroid gland: the importance of the clinical scenario. Appl Immunohistochem Mol Morphol 2019; 27: 726-731

25. Srilatha PS, Das J, Monappa V. Oncocytic variant of medullary thyroid carcinoma - a case report. Iran J Pathol 2019; 14: 83-86.

26. Domagala P, Domagala W. Nuclear CK19-immunopositive pseudoinclusions as a new additional objective diagnostic feature of papillary thyroid carcinoma. Pol J Pathol 2020; 71: 1-6.

27. Tastekin E, Keskin E, Can N, et al. CD56, CD57, HBME1, CK19, Galectin-3 and p63 immunohistochemical stains in differentiating diagnosis of thyroid benign/malign lesions and NIFTP. Pol J Pathol 2019; 70: 286-294.

28. Ordóñez NG. Thyroid transcription factor-1 is a marker of lung and thyroid carcinomas. Adv Anat Pathol 2000; 7 : $123-127$

29. Saad MF, Ordonez NG, Guido JJ, Samaan NA. The prognostic value of calcitonin immunostaining in medullary carcinoma of the thyroid. J Clin Endocrinol Metab 1984; 59: 850-856.

30. Erickson LA, Lloyd RV. Practical markers used in the diagnosis of endocrine tumors. Adv Anat Pathol 2004; 11: 175-189.

31. Zajac JD, Penschow J, Mason T, et al. Identification of calcitonin and calcitonin gene-related peptide messenger ribonucleic acid in medullary thyroid carcinomas by hybridization histochemistry. J Clin Endocrinol Metab 1986; 62: 1037-1043.

32. Lloyd RV. Use of molecular probes in the study of endocrine diseases. Hum Pathol 1978; 18: 1199-1211.

33. Yao YZ. CEA in lung cancer: CEA immunohistochemical study of 150 cases. Zhonghua Jie He He Hu Xi Za Zhi 1990; 13: $133-189$ 
34. Cheng L, Yin R, Yu Y, et al. Prognostic value of carcinoembryonic antigen level in advanced lung adenocarcinoma. Int J Clin Exp Med 2019; 12: 5812-5819.

35. Liu H, Lin F. Application of immunohistochemistry in thyroid pathology. Arch Pathol Lab Med 2015; 139: 67-82.

36. Prameela CG, Ravind R, Sruthi K. Nonthyroidal metastatic lesion in thyroid: A missed diagnosis and a lesson learnt. J Cancer Res Ther 2019; 15: 665-668.

37. D’Antonio A, Addesso M, De Dominicis G, et al. Mucinous carcinoma of thyroid gland. Report of a primary and a metastatic mucinous tumour from ovarian adenocarcinoma with immunohistochemical study and review of literature. Virchows Arch 2007; 451: 847-851.

38. Rosenbaum JN, Guo Z, Baus RM, et al. INSM1: a novel immunohistochemical and molecular marker for neuroendocrine and neuroepithelial neoplasms. Am J Clin Pathol 2015; 144: 579-591.

39. Khan A, Tischler AS, Patwardhan NA, DeLellis RA. Calcitonin immunoreactivity in neoplastic and hyperplastic parathyroid glands: an immunohistochemical study. Endocr Pathol 2003; 14: 249-255.

40. Li J, Chen W, Liu A. Clinincopathologic features of parathyroid carcinoma: a study of 11 cases with review of literature. Zhonghua Bing Li Xue Za Zhi 2014; 43: 296-300.

41. Hodgson A, Pakbaz S, Tayyari F, et al. Diagnostic pitfall: parathyroid carcinoma expands the spectrum of calcitonin and calcitonin gene-related peptide expressing neuroendocrine neoplasms. Endocr Pathol 2019; 30: 168-172.

42. Bauer JL, Toluie S, Thompson LDR. Metastases to the parathyroid glands: a comprehensive literature review of 127 reported cases. Head Neck Pathol 2018; 12: 534-541.

43. Tomita T. Immunocytochemical staining patterns for parathyroid hormone and chromogranin in parathyroid hyperplasia, adenoma, and carcinoma. Endocr Pathol 1999; 10: 145-156.

44. Erickson LA, Mete O. Immunohistochemistry in diagnostic parathyroid pathology. Endocr Pathol 2018; 29: 113-129.

45. Betts G, Beckett E, Nonaka D. GATA3 shows differential immunohistochemical expression across thyroid and parathyroid lesions. Histopathology 2014; 65: 288-290.

46. Duan K, Mete O. Algorithmic approach to neuroendocrine tumors in targeted biopsies: Practical applications of immunohistochemical markers. Cancer Cytopathol 2016; 124: 871-884.

47. Miettinen M, Mc. Cue PA, Sarlomo-Rikala M, et al. GATA 3 A multispecific but potentially useful marker in surgical pathology - a systematic analysis of 2500 epithelial and non-epithelial tumors. Am J Surg Pathol 2014; 38: 13-22.

48. Mahalakshmi B, Baskaran R, Shanmugavadivu M, et al. Insulinoma-associated protein 1 (INSM1): a potential biomarker and therapeutic target for neuroendocrine tumors. Cell Oncol (Dordr) 2020; 43: 367-376.

49. Duan K, Mete O. Algorithmic approach to neuroendocrine tumors in targeted biopsies: Practical applications of immunohistochemical markers. Cancer Cytopathol 2016; 124: 871-884.

\title{
Address for correspondence
}

\author{
Sarah Adel Hakim \\ Abbasseya Square \\ Faculty of Medicine \\ Ain Shams University \\ 11561, Cairo, Egypt \\ Tel. +2 01229326455 \\ e-mail: sarahadel2003@yahoo.com
}

